\title{
EXISTENCE OF A FIXED POINT FOR NONEXPANSIVE MAPPINGS WITH CLOSED VALUES
}

\author{
J. T. MARKIN
}

\begin{abstract}
Fixed point existence and fixed point stability results are presented for nonexpansive mappings of a Banach space $B$ into the family of nonempty closed bounded convex subsets of $B$, where $B$ is assumed separable, strictly convex, and reflexive with a weakly continuous duality mapping.
\end{abstract}

The study of the existence of a fixed point for nonexpansive set valued mappings was initiated in [7] for Hilbert space and extended in [6] to Banach spaces satisfying Opial's condition and in [5] to strictly convex reflexive Banach spaces with weakly continuous duality mapping. All of these results have assumed the mappings have compact values. In Theorem 1 the nonexpansive mapping is assumed to have closed bounded convex values and existence of a fixed point is shown for separable strictly convex reflexive Banach spaces with weakly continuous duality mapping. This class of spaces includes separable Hilbert spaces and the $l_{p}$ spaces, $1<p<\infty$.

The family of nonempty closed bounded convex subsets of a Banach space $B$ is denoted by $K(B)$. Let $D$ denote the Hausdorff metric defined on the closed bounded subsets of $B$, which is generated by the norm $\|\cdot\|$ of $B$. A mapping $F$ of $B$ into $K(B)$ is nonexpansive if $D(F(x), F(y)) \leqslant\|x-y\|$ for $x, y \in B$.

A mapping $J$ of a Banach space $B$ into its dual $B^{*}$ is a duality mapping if $(x, J(x))=\|x\|\|J(x)\|$ and $\|J(x)\|=\mu(\|x\|)$ for $x \in B$, where $\mu$ is a nonnegative nondecreasing function on $R^{1}$ with $\mu(0)=0$. A duality mapping $J$ is said to be weakly continuous if it is continuous from $B$ with the weak topology into $B^{*}$ with the weak*-topology. Weak convergence of a sequence $\left\{x_{i}\right\}$ to a point $x$ is denoted by $x_{i} \rightarrow x$.

A mapping $F$ of a Banach space $B$ into itself is $J$-monotone provided for any pair $x, y \in B$ and $x_{1} \in F(x)$ there is a $y_{1} \in F(y)$ such that $\left(x_{1}-y_{1}\right.$, $J(x-y)) \geqslant 0$, where $J$ is a duality mapping on $B$.

For any mapping $F$ of $B$ into the nonempty subsets of $B$ and any subset $C$ of $B, F(C)$ denotes $\cup_{x \in C} F(x)$. A point $y \in B$ is a fixed point of $F$ if $y \in F(y)$.

Received by the editors October 6, 1975 and, in revised form, February 24, 1976.

AMS (MOS) subject classifications (1970). Primary 47H10; Secondary 54C60.

Key words and phrases. Fixed point, set valued map, monotone map.

O American Mathematical Society 1976 
The following two lemmas extend similar results in [5] for compact valued mappings.

LEMMA 1. Let $B$ be a strictly convex reflexive Banach space with a weakly continuous duality mapping $J$, and $F$ a continuous mapping of $B$ with the norm topology into $K(B)$ with the Hausdorff metric $D$. If for a given pair $x, x_{1} \in B$ and any $y \in B$ there is a $y_{1} \in F(y)$ such that $\left(x_{1}-y_{1}, J(x-y)\right) \geqslant 0$, then $x_{1} \in F(x)$.

Proof. Let $x, x_{1}$. be elements of $B$ such that for any $y \in B$ there is $y_{1} \in F(y)$ satisfying $\left(y_{1}-x_{1}, J(y-x)\right) \geqslant 0$. Suppose $x_{1} \notin F(x)$. Since $F(x)$ is weakly compact and convex there is a continuous linear functional $w$ strictly separating $x_{1}$ and $F(x)$; i.e., $\left(x_{1}, w\right)<(z, w)$ for $z \in F(x)$. Ko [5] has shown that if $B$ is reflexive then a weakly continuous duality mapping maps $B$ onto $B^{*}$ and therefore $w=J(u)$ for some $u \in B$. Hence,

$$
\left(x_{1}, J(u)\right)<(z, J(u))
$$

for $z \in F(x)$.

Setting $u_{n}=x-u / n, n=1,2, \ldots$, there is by assumption for each $u_{n}$ a $z_{n} \in F\left(u_{n}\right)$ such that

$$
\left(x_{1}-z_{n}, J\left(x-u_{n}\right)\right)=\left(x_{1}-z_{n}, J(u / n)\right) \geqslant 0 .
$$

By a result of Browder [3] the strict convexity of the norm of $B$ implies that $J(u / n)=J(u) / n$. Inequality (2) can then be written as

$$
\left(x_{1}-z_{n}, J(u)\right) \geqslant 0
$$

for each $n$.

By the continuity of $F, D\left(F\left(u_{n}\right), F(x)\right)$ tends to 0 and therefore we may assume that $\left\{z_{n}\right\}$ converges weakly to a point $z_{0}$ and that there is a sequence $\left\{y_{n}\right\}, y_{n} \in F(x)$ for which $\lim _{n \rightarrow \infty}\left\|z_{n}-y_{n}\right\|=0$, where $y_{n} \rightarrow y_{0} \in F(x)$. We assert that $z_{0} \in F(x)$ so that by inequality (3), $\left(x_{1}, J(u)\right) \geqslant\left(z_{0}, J(u)\right)$, contradicting (1). Indeed, if $z_{0} \notin F(x)$ there is a continuous linear functional $v$ such that $\left(z_{0}, v\right)<\left(y_{0}, v\right)$ and hence

$$
0>\left(z_{0}-y_{0}, v\right)=\left(z_{0}-z_{n}, v\right)+\left(z_{n}-y_{n}, v\right)+\left(y_{n}-y_{0}, v\right) .
$$

The right side of the latter equality tends to 0 , which is not possible.

LEMMA 2. Let $B$ be a separable strictly convex reflexive Banach space with weakly continuous duality mapping $J, C$ a weakly compact subset of $B$, and $F a$ continuous J-monotone mapping of $B$ into $K(B)$ with the Hausdorff metric $D$. Then $F(C)$ is closed.

Proof. Let $v_{0}$ lie in the closure of $F(C)$. Then there is a sequence $\left\{v_{i}\right\}$ such that $\lim _{i \rightarrow \infty} v_{i}=v_{0}$, where $v_{i} \in F\left(u_{i}\right), u_{i} \in C$ and, by weak compactness of $C$, it is assumed that $u_{i} \rightarrow u_{0} \in C$. The assumption that $v_{0} \notin F(C)$ will be shown to lead to a contradiction. 
For some $x \in B$ it must be the case that there is a $\delta>0$ such that

$$
\left(z-v_{0}, J\left(x-u_{0}\right)\right)<-\delta
$$

for each $z \in F(x)$; for otherwise Lemma 1 would imply that $v_{0} \in F\left(u_{0}\right)$. For each nonnegative integer $j$ let $B_{j}=F(x)-v_{j}$. Since $v_{j} \rightarrow v_{0}$, the sequence $\left\{B_{j}\right\}$ converges to $B_{0}$ in the Hausdorff metric $D$.

Choose a closed ball $S$ in $B$ which contains the sets $\left\{B_{j}\right\}, j=0,1, \ldots$ By the reflexivity of $B$ the ball $S$ is weakly compact and by [4] the weak topology on $S$ is metrizable. Metrizing the weak topology on $S$ by

$$
d(a, b)=\sum_{n=1}^{\infty} \frac{1}{2^{n}} \frac{\left|x_{n}(a-b)\right|}{1+\left|x_{n}(a-b)\right|}
$$

where $\left\{x_{n}\right\}$ is a countable dense subset of the unit ball of $B^{*}$ and $a, b \in S$, it is easily seen that $\left\{B_{j}\right\}$ converges to $B_{0}$ in the Hausdorff metric $H$ generated by $d$. Indeed,

$$
d(a, b) \leqslant \sum_{n=1}^{\infty} \frac{1}{2^{n}} \frac{\|a-b\|}{1+\|a-b\|} \leqslant\left(\sum_{n=1}^{\infty} \frac{1}{2^{n}}\right)\|a-b\|
$$

implying that

$$
H(A, B) \leqslant\left(\sum_{n=1}^{\infty} \frac{1}{2^{n}}\right) D(A, B),
$$

where $A, B$ are weakly closed subsets of $S$.

Define the functionals $\left\{L_{i}\right\}$ on $B$ by $L_{i}(y)=\sup _{j \geqslant i}\left(y, J\left(x-u_{j}\right)\right), y \in B$. Since the functionals $\left\{\left(\cdot, J\left(x-u_{j}\right)\right)\right\}$ are linear and, hence, convex, each $L_{i}$ is a convex functional [2].

Let the function $L$ denote an arbitrary member of $\left\{L_{i}\right\}$. We claim that $L$ is continuous on $S$ with the weak relative topology. Since $L$ is bounded on any norm bounded subset of $B$, assume that $L(y) \leqslant K$ for any $y \in S$. Let $N(0, \delta)$ be an origin centered open ball of radius $\delta$ in the weak metric topology on $S$. For $\alpha \in[0,1)$ it is easily seen that $\alpha N(0, \delta) \subseteq N(0, \delta)$. If $y \in \alpha N(0, \delta)$ then there is a $z \in N(0, \delta)$ for which $y=\alpha z$ and therefore $(y / \alpha) \in N(0, \delta)$. By the convexity of $L$,

$$
L((1-\alpha) 0+\alpha(y / \alpha)) \leqslant(1-\alpha) L(0)+\alpha L(y / \alpha) .
$$

Since $L(0)=0, L(y) \leqslant \alpha L(y / \alpha) \leqslant \alpha K$. Hence, in the limit as $y$ approaches 0 in the weak topology $L(y) \leqslant L(0)$, which proves that $L$ is upper semicontinuous at 0 in the weak topology on $S$. Continuity of $L$ at 0 follows from the lower semicontinuity of the supremum of continuous functions and the definition of $L$. For any other point $y \in S$ appropriate translations reduce the problem to the case just considered where $y=0$ and $L(y)=0$.

Since $F$ is $J$-monotone, for each $u_{j}$ there is a $z_{j} \in F(x)$ such that $\left(z_{j}-v_{j}, J\left(x-u_{j}\right)\right) \geqslant 0$. Thus, for each $j \geqslant i, \sup _{y \in B_{j}} L_{i}(y) \geqslant 0$. The maximum theorem in [1] implies that 


$$
\lim _{j \rightarrow \infty} \sup _{y \in B_{j}} L_{i}(y)=\sup _{y \in B_{0}} L_{i}(y) \geqslant 0 \quad \text { for } i=1,2, \ldots
$$

For each positive integer $i$ let $A_{i}=\left\{y \in B_{0}: L_{i}(y) \geqslant-1 / i\right\}$. If $y \in A_{i+1}$ then, by the definition of the $L_{i}, L_{i}(y) \geqslant L_{i+1}(y) \geqslant-1 /(i+1)>-1 / i$, and therefore, $y \in A_{i}$. Thus, $A_{i+1} \subseteq A_{i}$ and, since the $A_{i}$ are weakly closed subsets of the weakly compact set $B_{0}$, there is a point $y_{0} \in \cap_{i=1}^{\infty} A_{i}$. By the definition of $B_{0}, y_{0}=z_{0}-v_{0}$ where $z_{0} \in F(x)$, and by (5) for some subsequence $\left\{u_{k}\right\}$ of $\left\{u_{i}\right\},\left(z_{0}-v_{0}, J\left(x-u_{k}\right)\right) \geqslant-1 / k$. Taking the limit in the latter inequality we have $\left(z_{0}-v_{0}, J\left(x-u_{0}\right)\right) \geqslant 0$, which contradicts (4).

THEOREM 1. Let $B$ be a separable strictly convex reflexive Banach space with a weakly continuous duality mapping $J$, and $C$ a closed bounded convex subset of $B$. If $G$ is a nonexpansive mapping of $B$ into $K(B)$ with the Hausdorff metric $D$, which maps $C$ into itself, then $G$ has a fixed point in $C$.

Proof. Assume without loss of generality that $0 \in C$ [5]. The proof consists of showing that 0 is in the closure of $(I-G)(C)$ and that $I-G$ is $J$ monotone. The theorem then follows by Lemma 2 .

Let $\left\{k_{i}\right\} \subseteq[0,1)$ be a sequence which converges to 1 , and consider the sequence of mappings $\left\{k_{i} G\right\}$ of $C$ into $K(C)$. By a result of Nadler [8] each $k_{i} G$ has a fixed point $x_{k_{i}} \in C$. Since $x_{k_{i}} \in k_{i} G\left(x_{k_{i}}\right)$ we have $x_{k_{i}}=k_{i} y_{k_{i}}$, where $y_{k_{i}} \in G\left(x_{k_{i}}\right)$. Therefore,

$$
\inf _{y \in G\left(x_{k_{i}}\right)}\left\|y-x_{k_{i}}\right\| \leqslant\left\|x_{k_{i}}-y_{k_{i}}\right\| \leqslant\left(1-k_{i}\right)\left\|y_{k_{i}}\right\|
$$

and the last term tends to 0 . This shows that 0 is in the closure of $(I-G)(C)$.

$G$ being nonexpansive with closed convex values, given any $y \in B$ and $y_{1} \in G(y)$, there is a closest point $x_{1} \in G(x)$ to $y_{1}$ such that $\left\|x_{1}-y_{1}\right\|$ $\leqslant\|x-y\|$. It follows that

$$
\left(\left(x-x_{1}\right)-\left(y-y_{1}\right), J(x-y)\right) \geqslant\left(\|x-y\|-\left\|x_{1}-y_{1}\right\|\right)\|J(x-y)\| \geqslant 0,
$$

and hence $I-G$ is $J$-monotone. Applying Lemma 2 we have $0 \in(I-G)$ - $(C)$; i.e., there is an $x \in C$ such that $x \in G(x)$.

THEOREM 2. Let $B$ be a separable strictly convex reflexive Banach space with a weakly continuous duality mapping $J$, and $C$ a closed bounded convex subset of $B$. Assume that $\left\{G_{i}\right\}$ is a sequence of nonexpansive mappings of $B$ into $K(B)$ with the Hausdorff metric, which converges pointwise to a nonexpansive mapping $G_{0}$ and maps $C$ into itself. If $x_{i} \in C$ is a fixed point of $G_{i}, i=1,2, \ldots$, and $x_{i} \rightarrow x_{0}$ then $x_{0}$ is a fixed point of $G_{0}$.

Proof. As in the proof of Theorem 1 the mappings $I-G_{i}, i=0,1, \ldots$, are $J$-monotone. Since $x_{i}$ is a fixed point of $G_{i}, 0 \in\left(I-G_{i}\right)\left(x_{i}\right)$ for $i=1,2$, $\ldots$, and by $J$-monotonicity for each $v \in B$ there is a $v_{i} \in\left(I-G_{i}\right)(v)$ for which

$$
\left(v_{i}-0, J\left(v-x_{i}\right)\right) \geqslant 0, \quad i=1,2, \ldots
$$


Define the sequence of functionals $\left\{L_{i}\right\}$ on $B$ by

$$
L_{i}(y)=\sup _{j \geqslant i}\left(y, J\left(v-x_{j}\right)\right) .
$$

As in the proof of Lemma 2 the $\left\{L_{i}\right\}$ are continuous on $S$ with the weak topology. Defining the sequence of weakly compact convex subsets $\left\{B_{j}\right\}$ of $B$ by $B_{j}=\left(I-G_{j}\right)(v)$, the pointwise convergence of the $\left\{G_{j}\right\}$ implies that $\left\{B_{j}\right\}$ converges to $B_{0}=\left(I-G_{0}\right)(v)$ in the Hausdorff metric $D$, and therefore, as shown in the proof of Lemma 2 , the $\left\{B_{j}\right\}$ converge to $B_{0}$ in the Hausdorff metric $H$ generated by the weak metric topology on any ball containing the $\left\{B_{j}\right\}$. Inequality (6) implies that for each $i, \sup _{y \in B_{j}} L_{i}(y) \geqslant 0$ for $j \geqslant i$.

Thus, the sequences $\left\{L_{i}\right\}$ and $\left\{B_{j}\right\}$ satisfy the same conditions as in the proof of Lemma 2, and therefore there is a point $y_{0} \in B_{0}$ such that $\left(y_{0}-0\right.$, $\left.J\left(v-x_{0}\right)\right) \geqslant 0$. The point $v \in B$ was arbitrary and so by Lemma 1,0 $\in\left(I-G_{0}\right)\left(x_{0}\right)$ and $x_{0}$ is a fixed point of $G_{0}$.

\section{REFERENCES}

1. C. Berge, Espaces topologiques; Fonctions multivoques, Collection Universitaire de Mathématiques, vol. III, Dunod, Paris, 1959. MR 21 \#4401.

2. N. Bourbaki, Espaces vectoriels topologiques. I, II, Actualités Sci. Indust., nos. 1189, 1229, 1230, Hermann, Paris, 1953, 1955. MR 14, 880; 17, 1109.

3. F. E. Browder, On a theorem of Beurling and Livingston, Canad. J. Math. 17 (1965), 367-372. MR 31 \# 595.

4. N. Dunford and J. T. Schwartz, Linear operators. I: General theory, Pure and Appl. Math., vol. 7, Interscience, New York, 1958. MR 22 \#8302.

5. H. Ko, A fixed point theorem for point-to-set mappings, Dept. of Math., New Asia College, Chinese Univ. of Hong Kong, Kowloon, Hong Kong, 1972.

6. E. Lami Dozo, Multivalued nonexpansive mappings and Opial's condition, Proc. Amer. Math. Soc. 38 (1973), 286-292. MR 46 \#9816.

7. J. T. Markin, A fixed point theorem for set valued mappings, Bull. Amer. Math. Soc. 74 (1968), 639-640. MR 37 \#3409.

8. S. B. Nadler Jr., Multi-valued contraction mappings, Pacific J. Math. 30 (1969), 475-488. MR $40 \# 8035$.

Kaman Sciences Corporation, 1500 Garden of the Gods Road, Colorado Springs, Colorado 80907 